\title{
BIFURCATING STEADY-STATE SOLUTIONS OF THE DISSIPATIVE QUASI-GEOSTROPHIC EQUATION IN LAGRANGIAN FORMULATION
}

\author{
ZHI-MIN CHEN
}

\begin{abstract}
It is shown that the non-homogeneous dissipative quasi-geostrophic equation

$$
\frac{\partial \theta}{\partial t}+u \cdot \nabla \theta+\kappa(-\Delta)^{\alpha} \theta=\sin x_{2}, \quad u=\left(-\partial_{x_{2}}, \partial_{x_{1}}\right)(-\Delta)^{-\beta / 2} \theta
$$

with $\alpha=0$ and $\beta>1$ losses stability at a critical value $\kappa_{c}>0$ and this instability gives rise to a circle of steady-state solutions.

Keywords: quasi-geostrophic equation, steady-state bifurcation, Lagrange formulation
\end{abstract}

Mathematics Subject Classification: 35B32, 35Q35, 86A10

\section{INTRODUCTION}

A surface quasi-geostrophic flow is the first order approximation of a geostrophic flow with respect to the quasi-geostrophic approximation under a small Rossby number [27]. Instability examination of quasi-geostrophic equation is significant important in the understanding of geophysical variabilities [16]. From the mathematical formulation of [10], the energy dissipation controlled by the fractional Laplacian $\kappa(-\Delta)^{\alpha}$ is applied to the quasi-geostrophic flow [11, 32] and the homogeneous flow is thus governed by the dissipative quasi-geostrophic equation

$$
\frac{\partial \theta}{\partial t}+u \cdot \nabla \theta+\kappa(-\Delta)^{\alpha} \theta=0, \quad u=\left(-\partial_{x_{2}}, \partial_{x_{1}}\right)(-\Delta)^{-1 / 2} \theta
$$

for $0 \leq \alpha \leq 1$, the dissipative parameter $\kappa>0$, the gradient operator $\nabla$ and the Laplacian $\Delta=\nabla \cdot \nabla$.

Equation (1) provides an interesting simple model that exhibits a number of nonlinear and dissipative characters of the three-dimensional Navier-Stokes equations, and hence has been studied extensively since the late 1990s. Chae and Lee [2], Chen et al. [4] and $\mathrm{Wu}$ [33] derived the existence of local regular solutions and global small solutions in the framework of Besov spaces for $\alpha>$. This well-posedness problem was also examined by Córdoba and Córdoba [13] in $H^{m}$ spaces for $\alpha>0$. For $\alpha>\frac{1}{2}$ (the subcritical case), it is well known (see [11, 18]) that a smooth initial function $\theta_{0}$ with a compact support leads to a global regular solution. For the critical case $\alpha=\frac{1}{2}$, similar to the three-dimensional Navier-Stokes equations, the nonlinear term is difficult to be controlled by the dissipative term for large solutions. However, due to the validity of the maximal principle for (1), the global existence of regular solution was obtained by Kiselev et al. [21] (see also Caffarelli and Vasseur [1]). When $0<\alpha<\frac{1}{2}$ (the supercritical case), the dissipation is too 
weak to ensure the global existence of large regular solutions. Therefore for the efforts on finite blow up examinations, we refer to Kiselev [19, 20] and references therein. However, the global existence is obtainable if additional conditions are applied (see, for example, Constantin and $\mathrm{Wu}$ [12] and Dong and Pavlovic [15]). One may also refer to Chen and Xiong [8] for computational steady-state bifurcation results of Charney and DeVore quasi-geostrophic equation and to Kato and Ponce [22], Ju [18], Danchin [14] and Chen and Chen [3] for commutator estimates applicable to equation (1).

Note that the dissipative quasi-geostrophic equation (1) defines a flow without external forcing. As a result of the energy dissipation, the flow motion decays for $\alpha>0$ (see Constantin and Wu [11] and Schonbek and Schonbek [28]). The also implies the nonlinear stability of the trivial steady-state solution $\theta=0$ and then the nonlinear stability for the quasi-geostrophic equation driven by a small steady-state external forcing. However, when the external forcing is not small and the strength of nonlinear convection is overtaking the strength of the energy dissipation by decreasing the parameter $\kappa$, nonlinear instability arises and steadystate bifurcation may occur.

In the present study, the dissipative quasi-geostrophic flow is driven by the typical thermal forcing $f=\sin x_{2}$ (see Iserley and Sheremet [17], Pedlosky [27] and Veronis [30]) and thus is determined by the non-homogeneous dissipative quasi-geostrophic equations

$$
\frac{\partial \theta}{\partial t}+u \cdot \nabla \theta+\kappa(-\Delta)^{\alpha} \theta=\sin x_{2}, \quad u=\left(-\partial_{x_{2}}, \partial_{x_{1}}\right)(-\Delta)^{-1 / 2} \theta
$$

Here the solution $\theta$ is spatially periodic in the domain $[0,2 \pi b] \times[0,2 \pi]$ for $0<b \leq 1$ and (2) admits a basic steady-state solution $\hat{\theta}=\frac{1}{\kappa} \sin x_{2}$.

It has been shown in [7] that $\hat{\theta}$ is globally nonlinear stable for $b=1$ and $\hat{\theta}$ bifurcates into a circle of steady-state solutions as $\kappa$ increasing across a critical value $\kappa_{c}>0$ for $\frac{1}{2} \leq b<1$. The bifurcating solutions are constructed by the amplitude expansion technique, which is only applicable to the case $\alpha \geq \frac{1}{2}$. It was expected in [7] to extend this bifurcation result to the lower limit situation $\alpha=0$ of the strong critical case. Unfortunately, we have not obtained an available estimate to ensure the compactness required in the bifurcation analysis.

However, the steady-state bifurcation result can be obtained if we generalize the dissipative quasi-geostrophic equation (2) by modifying the vector field with $u=\left(-\partial_{x_{2}}, \partial_{x_{1}}\right)(-\Delta)^{-\beta / 2} \theta$ as discussed by Constantin et al. [9], Kiselev [20] and Yamazaki [34].

More precisely, the main result of this paper reads as follows:

Theorem 1.1. For $\frac{3}{4} \leq b<1, \beta>1$ and $2 /(\beta-1)<p<\infty$, the dissipative quasi-geostrophic equation

$$
\frac{\partial \theta}{\partial t}+u \cdot \nabla \theta+\kappa \theta=\sin x_{2}, \quad u=\left(-\partial_{x_{2}}, \partial_{x_{1}}\right)(-\Delta)^{-\beta / 2} \theta
$$

admits a positive critical value $\kappa_{c}$ satisfying

$$
\kappa_{c}^{4}<\frac{\beta}{4} b^{4-\beta}\left(1-b^{\beta}\right)
$$

and a classical steady-state solution $\theta_{\kappa, \phi} \in H_{p}^{1+\beta}\left(\Omega_{b}\right)$ for $0 \leq \phi<2 \pi$ bifurcating from the basic steady-state solution $\hat{\theta}_{0}=\frac{1}{\kappa_{c}} \sin x_{2}$ when $\kappa$ varies across $\kappa_{c}$. 
Here $H_{p}^{\tau}\left(\Omega_{b}\right)$ denotes the fractional Sobolev space on the two-dimensional periodic domain $\Omega_{b}=R^{2} /(2 \pi b Z \times 2 \pi Z)$ :

$$
H_{p}^{\tau}\left(\Omega_{b}\right)=\left\{f \in L_{p}\left(\Omega_{b}\right) ; \int_{0}^{2 \pi b} \int_{0}^{2 \pi} f d x_{1} d x_{2}=0,\|f\|_{H_{p}^{\tau}}<\infty\right\}
$$

with the norm

$$
\|f\|_{H_{p}^{\tau}}=\|f\|_{L_{p}}+\left\|(-\Delta)^{\frac{\tau}{2}} f\right\|_{L_{p}}
$$

and the $L_{p}$ norm

$$
\|f\|_{L_{p}}=\left(\int_{0}^{2 \pi b} \int_{0}^{2 \pi}|f|^{p} d x_{1} d x_{2}\right)^{1 / p} .
$$

The fractional Laplacian $(-\Delta)^{\tau}$ is in the following sense:

$$
(-\Delta)^{\tau} \mathrm{e}^{-\mathrm{i}\left(m b x_{1}+n x_{2}\right)}=\left((m b)^{2}+n^{2}\right)^{\tau} \mathrm{e}^{-\mathrm{i}\left(m b x_{1}+n x_{2}\right)} .
$$

The instability property of (3) exhibits the coexistence of a circle of steady-state solutions $\theta_{\kappa, \phi}$ for $0 \leq \phi<2 \pi$ and its profile is displayed in Figure 1. For a circle bifurcation theory and the stability of bifurcating steady-state flows, one may refer to Ma and Wang [24]. The present steady-state bifurcation analysis is developed from the author [5] by using a Lagrange formulation technique and the traditional steady-state bifurcation theorem given by Krasnoselskii [23].

This paper is organized as follows. In Section 2, a Lagrangian formulation approach is developed from [5] so that the dissipative quasi-geostrophic flow in a neighborhood of the basic flow $\hat{\theta}$ in fractional Sobolev spaces is estimated and the compactness required by the bifurcation analysis is obtained. Section 3 exhibits the 1 grangian $\mathrm{fc}$ $[5,6,7,35$, in Section. bility deriv assumptior in Krasnos

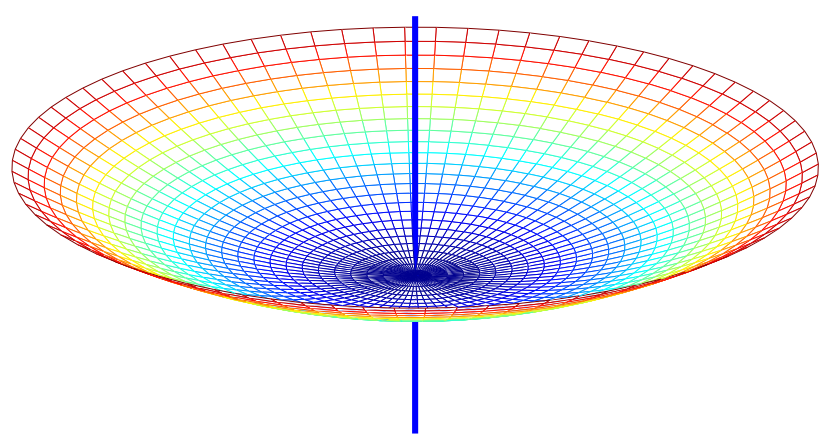

Figure 1. The nonlinear instability profile for a circle of steadystate flows branching off the basic steady-state flow as $\kappa$ varying across the critical value $\kappa_{c}$. 
2. LAgRANGIAN FORMULATION FOR THE STATIONARY EQUATION OF (3)

For convenience, we employ the notation $\theta^{\prime}=\kappa \theta$ and $u^{\prime}=\kappa u$ to rewrite the stationary equation of (3) as

$$
u \cdot \nabla \theta+\kappa^{2} \theta=\kappa^{2} \sin x_{2}, u=\left(-\partial_{x_{2}}, \partial_{x_{1}}\right)(-\Delta)^{-\beta / 2} \theta,
$$

where the prime symbol have been omitted. The corresponding steady-state solution $\hat{\theta}$ of $(3)$ becomes

$$
\theta_{0}=\sin x_{2}
$$

the basic solution of $(6)$.

The divergence free vector field $u$ in (6) is in the following Banach space

$$
H_{\infty}^{1}\left(\Omega_{b}\right)=\left\{v \in L_{\infty}\left(\Omega_{b}\right) ;\|v\|_{H_{\infty}^{1}}=\|v\|_{L_{\infty}}+\|\nabla v\|_{L_{\infty}}<\infty\right\} .
$$

Since the bifurcation in Theorem 1.1 is local, we consider the flow motion when $u$ is close to the steady-state vector field

$$
u_{0}=\left(-\cos x_{2}, 0\right)=\left(-\partial_{x_{2}}, \partial_{x_{1}}\right)(-\Delta)^{-\frac{\beta}{2}} \theta_{0} .
$$

For a trajectory $y=\left(y_{1}, y_{2}\right)$ initiating from a particle $x=\left(x_{1}, x_{2}\right)$ in the fluid domain, we have the Lagrangian formulation

$$
\left\{\begin{aligned}
-\frac{\partial}{\partial s} y(x, s) & =u(y(x, s)), \quad s>0, \\
y(x, 0) & =x \in \Omega_{b} .
\end{aligned}\right.
$$

Applying the gradient operator $\nabla=\left(\partial_{x_{1}}, \partial_{x_{2}}\right)$ to $(7)$, we have

$$
\begin{aligned}
-\frac{\partial}{\partial s} \nabla y(x, s) & =\nabla y \cdot \nabla_{y} u(y(x, s)), s>0 \\
\nabla y(x, 0) & =I .
\end{aligned}
$$

for the $2 \times 2$ identity matrix $I$ and the gradient $\nabla_{y}=\left(\partial_{y_{1}}, \partial_{y_{2}}\right)$.

The flow motion around the basic stead-state vector field $u_{0}$ is described by the result.

Lemma 2.1. Let $u \in H_{\infty}^{1}\left(\Omega_{b}\right)$ so that $\left\|\nabla u-\nabla u_{0}\right\|_{L_{\infty}} \leq \frac{1}{2}$. Then the estimate

$$
\|\nabla y(\cdot, s)\|_{L_{\infty}} \leq \sqrt{2}(1+s) e^{2 s \sqrt{\left\|\nabla u-\nabla u_{0}\right\|_{L_{\infty}}}}
$$

holds true for $s \geq 0$.

To show the validity of $(10)$ with $u=\left(u_{1}, u_{2}\right)$, we see that

$$
\left\|\partial_{y_{2}} u_{1}(y)-\sin y_{2}\right\|_{L_{\infty}} \leq\left\|\nabla u-\nabla u_{0}\right\|_{L_{\infty}} .
$$

and for $(i, j) \neq(2,1)$

$$
\left\|\partial_{y_{i}} u_{j}(y)\right\|_{L_{\infty}} \leq\left\|\nabla u-\nabla u_{0}\right\|_{L_{\infty}} .
$$


By an elementary calculation and the use of (8), we have

$$
\begin{aligned}
& \frac{\partial}{\partial s}\left(\begin{array}{ll}
\left\|\partial_{x_{1}} y_{1}\right\|_{L_{\infty}} & \left\|\partial_{x_{2}} y_{1}\right\|_{L_{\infty}} \\
\left\|\partial_{x_{1}} y_{2}\right\|_{L_{\infty}} & \left\|\partial_{x_{2}} y_{2}\right\|_{L_{\infty}}
\end{array}\right) \\
& \leq\left(\begin{array}{cc}
\left\|\partial_{y_{1}} u_{1}\right\|_{L_{\infty}} & \left\|\partial_{y_{2}} u_{1}\right\|_{L_{\infty}} \\
\left\|\partial_{y_{1}} u_{2}\right\|_{L_{\infty}} & \left\|\partial_{y_{2}} u_{2}\right\|_{L_{\infty}}
\end{array}\right)\left(\begin{array}{cc}
\left\|\partial_{x_{1}} y_{1}\right\|_{L_{\infty}} & \left\|\partial_{x_{2}} y_{1}\right\|_{L_{\infty}} \\
\left\|\partial_{x_{1}} y_{2}\right\|_{L_{\infty}} & \left\|\partial_{x_{2}} y_{2}\right\|_{L_{\infty}}
\end{array}\right) \\
& \leq\left(\begin{array}{cc}
\sigma & 1+\sigma \\
\sigma & \sigma
\end{array}\right)\left(\begin{array}{cc}
\left\|\partial_{x_{1}} y_{1}\right\|_{L_{\infty}} & \left\|\partial_{x_{2}} y_{1}\right\|_{L_{\infty}} \\
\left\|\partial_{x_{1}} y_{2}\right\|_{L_{\infty}} & \left\|\partial_{x_{2}} y_{2}\right\|_{L_{\infty}}
\end{array}\right)
\end{aligned}
$$

for $\sigma=\left\|\nabla u-\nabla u_{0}\right\|_{L_{\infty}}$. Without loss of generality, we may assume $\sigma>0$. The combination of the previous equation and the initial identity condition (9) implies

$$
\begin{aligned}
& \left(\begin{array}{cc}
\left\|\partial_{x_{1}} y_{1}\right\|_{L_{\infty}} & \left\|\partial_{x_{2}} y_{1}\right\|_{L_{\infty}} \\
\left\|\partial_{x_{1}} y_{2}\right\|_{L_{\infty}} & \left\|\partial_{x_{2}} y_{2}\right\|_{L_{\infty}}
\end{array}\right) \\
& \leq \exp \left(s\left(\begin{array}{ll}
\sigma & 1+\sigma \\
\sigma & \sigma
\end{array}\right)\right) \\
& =\left(\begin{array}{cc}
\frac{\sqrt{\sigma^{2}+\sigma}}{2 \sigma} & \frac{\sqrt{\sigma^{2}+\sigma}}{2 \sigma} \\
\frac{1}{2} & -\frac{1}{2}
\end{array}\right)\left(\begin{array}{lr}
\mathrm{e}^{s\left(\sigma+\sqrt{\sigma^{2}+\sigma}\right)} & 0 \\
0 & \mathrm{e}^{s\left(\sigma-\sqrt{\sigma^{2}+\sigma}\right)}
\end{array}\right)\left(\begin{array}{rr}
\frac{\sigma}{\sqrt{\sigma^{2}+\sigma}} & 1 \\
\frac{\sigma}{\sqrt{\sigma^{2}+\sigma}} & -1
\end{array}\right),
\end{aligned}
$$

and hence, for the eigenvalues $\lambda_{1}=\sigma+\sqrt{\sigma^{2}+\sigma}$ and $\lambda_{2}=\sigma-\sqrt{\sigma^{2}+\sigma}$,

$$
\begin{aligned}
\|\nabla y(\cdot, s)\|_{L_{\infty}}^{2} & \leq \frac{2\left(\mathrm{e}^{\lambda_{1} s}+\mathrm{e}^{\lambda_{2} s}\right)^{2}+\left(\frac{\sigma+1}{\sigma}+\frac{\sigma}{\sigma+1}\right)\left(\mathrm{e}^{\lambda_{1} s}-\mathrm{e}^{\lambda_{2} s}\right)^{2}}{4} \\
& \leq \frac{8 \mathrm{e}^{2 \lambda_{1} s}+\left(\frac{\sigma+1}{\sigma}+\frac{\sigma}{\sigma+1}\right)\left(\lambda_{1}-\lambda_{2}\right)^{2} s^{2} \mathrm{e}^{2 \lambda_{1} s}}{4} \\
& \leq\left(2+s^{2}\right) \mathrm{e}^{2 s\left(\sigma+\sqrt{\sigma^{2}+\sigma}\right)}
\end{aligned}
$$

This implies (10) due to the condition $\sigma \leq \frac{1}{2}$.

With the use of the Lagrangian formulation, the equation in Euler formulation can be written in the equation in Lagrangian formulation.

Lemma 2.2. Assume that $1 \leq p<\infty, \kappa>0, f \in H_{p}^{1}\left(\Omega_{b}\right)$ and $u \in H_{\infty}^{1}\left(\Omega_{b}\right)$. Then the operator $\kappa^{2}+u \cdot \nabla$ has a bounded inverse represented as

$$
\left(\kappa^{2}+u \cdot \nabla\right)^{-1} f=\int_{0}^{\infty} \mathrm{e}^{-s \kappa^{2}} f(y(x, s)) d s
$$

in the following sense

$$
\left\|\left(\kappa^{2}+u \cdot \nabla\right)^{-1} f\right\|_{L_{p}} \leq \frac{1}{\kappa^{2}}\|f\|_{L_{p}}
$$

Additionally, if $u$ is subject to the condition

$$
\left\|\nabla u-\nabla u_{0}\right\|_{L_{p}} \leq \frac{\kappa^{4}}{5},
$$

then there holds the estimate

$$
\left\|\nabla\left(\kappa^{2}+u \cdot \nabla\right)^{-1} f\right\|_{L_{p}} \leq \frac{16 \sqrt{2}\left(\kappa^{2}+1\right)}{\kappa^{4}}\|\nabla f\|_{L_{p}} .
$$


Proof. For $f \in H_{p}^{1}\left(\Omega_{b}\right)$, let $\varphi$ satisfy the equation

$$
\left(\kappa^{2}+u(x) \cdot \nabla\right) \varphi(x)=f(x) .
$$

By the Lagrange formulation (7), we rewrite the previous equation as

$$
\left(\kappa^{2}+u(y(x, s)) \cdot \nabla_{y}\right) \varphi(y(x, s))=f(y(x, s))
$$

for $s \geq 0$, and hence

$$
\left(\kappa^{2}-\frac{\partial y(x, s)}{\partial s} \cdot \nabla_{y}\right) \varphi(y(x, s))=f(y(x, s)),
$$

or, after the multiplication of the function $-\mathrm{e}^{-\kappa^{2} s}$ and the use of (7),

$$
\frac{\partial\left[\mathrm{e}^{-\kappa^{2} s} \varphi(y(x, s))\right]}{\partial s}=-\mathrm{e}^{-\kappa^{2} s} f(y(x, s)) .
$$

This gives the expression

$$
\varphi(x)=\int_{0}^{\infty} \mathrm{e}^{-s \kappa^{2}} f(y(x, s)) d s .
$$

It is readily seen that the kernel of the operator $\kappa^{2}+u \cdot \nabla$ is the set $\{0\}$. Thus (11) is valid.

Moreover, by the incompressible flow transformation property

$$
\operatorname{det}(\nabla y(x, s)) \equiv 1
$$

due to the divergence free condition $\nabla \cdot u=0$, we have the desired bounds

(20) $\left\|\left(\kappa^{2}+u \cdot \nabla\right)^{-1} f(x)\right\|_{L_{p}} \leq \int_{0}^{\infty} \mathrm{e}^{-s \kappa^{2}}\|f(y(x, s))\|_{L_{p}} d s=\frac{1}{\kappa^{2}}\|f\|_{L_{p}}$,

and, by (10) and (13),

$$
\begin{aligned}
& \left\|\nabla\left(\kappa^{2}+u \cdot \nabla\right)^{-1} f(x)\right\|_{L_{p}} \leq \int_{0}^{\infty} \mathrm{e}^{-s \kappa^{2}}\|\nabla f(y(x, s))\|_{L_{p}} d s \\
& \leq \int_{0}^{\infty} \mathrm{e}^{-s \kappa^{2}}\|\nabla y(x, s)\|_{L_{\infty}}\left\|\nabla_{y} f(y(x, s))\right\|_{L_{p}} d s \\
& \leq \int_{0}^{\infty} e^{-s \kappa^{2}} \sqrt{2}(1+s) \mathrm{e}^{s \kappa^{2} 2 / \sqrt{5}} d s\|\nabla f\|_{L_{p}} \\
& \leq \frac{\sqrt{2}}{\left(1-\frac{2}{\sqrt{5}}\right)^{4}}\left(\frac{1}{\kappa^{2}}+\frac{1}{\kappa^{4}}\right)\|\nabla f\|_{L_{p}} .
\end{aligned}
$$

This gives the desired estimate and the proof is complete.

As a consequence of Lemma 2.2, equation (6) becomes

$$
\theta(x)-\kappa^{2}\left(\kappa^{2}+u \cdot \nabla\right)^{-1} \theta_{0}=0, \quad u=\left(-\partial_{x_{2}}, \partial_{x_{1}}\right)(-\Delta)^{-\beta / 2} \theta,
$$

or

$$
\psi(x)-\kappa^{2}(-\Delta)^{-\beta / 2}\left(\kappa^{2}+u \cdot \nabla\right)^{-1} \psi_{0}=0, \quad u=\left(-\partial_{x_{2}}, \partial_{x_{1}}\right) \psi .
$$

for the stream function $\psi=(-\Delta)^{-\beta / 2} \theta$ and $\psi_{0}=\theta_{0}=\sin x_{2}$. 


\section{Linear instability}

Linearizing the operator $-\kappa^{2}(-\Delta)^{-\beta / 2}\left(\kappa^{2}+u \cdot \nabla\right)^{-1} \psi_{0}$ with respect to $\psi$ around $\psi_{0}$, we have the linear operator

$$
\begin{aligned}
M_{\kappa} \psi & =-(-\Delta)^{-\beta / 2}\left(\kappa^{2}+u_{0} \cdot \nabla\right)^{-1} u_{0} \cdot \nabla \psi \\
& =(-\Delta)^{-\beta / 2}\left(\kappa^{2}-\cos x_{2} \partial_{x_{1}}\right)^{-1} \cos x_{2} \partial_{x_{1}} \psi
\end{aligned}
$$

Therefore, we have the expression

$$
\psi(x)-\kappa^{2}\left(\kappa^{2}+u \cdot \nabla\right)^{-1} \psi_{0}=\psi-\psi_{0}+M_{\kappa}\left(\psi-\psi_{0}\right)+N(\psi, \kappa)
$$

for a nonlinear operator $N$.

Thus to show the bifurcation behaviour of (22), it is necessary to obtain the existence of a critical value $\kappa=\kappa_{c}$ and an eigenfunction $\psi$ so that

$$
\psi+M_{\kappa} \psi=0
$$

or

$$
\kappa^{2}(-\Delta)^{\beta / 2} \psi-\cos x_{2} \partial_{x_{1}}\left[(-\Delta)^{\beta / 2}-1\right] \psi=0 .
$$

Lemma 3.1. Let $\frac{3}{4} \leq b<1,0 \leq \phi<2 \pi, 1 \leq \beta$ and $1<p<\infty$. Assume that $m \neq 1$ is an integer. Then for any $\kappa>0$

$$
\text { ( } \left.\{\pi)=\sum_{n=-\infty}^{\infty} a_{n} \cos \left(m b x_{1}+\phi+n x_{2}+\frac{n \pi}{2}\right) \in H_{p}^{1+\beta}\left(\Omega_{b}\right) ; \psi+M_{\kappa} \psi=0\right\}=\{0\},
$$

and there exists a critical value $\kappa=\kappa_{c}$ so that

d(28) $\bigcup_{k=1}^{\infty}\left\{\psi=\sum_{n=-\infty}^{\infty} \frac{a_{n} \cos \left(b x_{1}+\phi+n x_{2}+\frac{n \pi}{2}\right)}{b\left[\left(b^{2}+n^{2}\right)^{\frac{\beta}{2}}-1\right]} \in H_{p}^{1+\beta}\left(\Omega_{b}\right) ;\left(1+M_{\kappa}\right)^{k} \psi=0\right\}=1$.

This result is obtained by a continued fraction technique developed from Chen et al. [6], Chen and Price [7] and Chen [5] and originated from Mishalkin and Sinai [25] and Yudovich [35].

Proof. Multiplying (26) by $\kappa^{-2}\left[(-\Delta)^{\frac{\beta}{2}}-1\right] \psi$ and integrating the resultant equation over the domain $\Omega_{b}$, we have the integral equation

$$
0=\int_{0}^{2 \pi / b} \int_{0}^{2 \pi}(-\Delta)^{\frac{\beta}{2}} \psi\left[(-\Delta)^{\frac{\beta}{2}} \psi-\psi\right] d x_{1} d x_{2},
$$

after taking integration by parts. The substitution of the function

$$
\psi=\sum_{n=-\infty}^{\infty} a_{n} \cos \left(b m x_{1}+\phi+n x_{2}+\frac{n \pi}{2}\right), \quad m \neq 1 .
$$

into (29) implies $a_{n} \equiv 0$ and hence (27) is verified. Here we have used the average condition

$$
\int_{\Omega_{b}} \psi d x_{1} d x_{2}=0
$$

implied in the space $H_{p}^{1}\left(\Omega_{b}\right)$ defined in (5) to confirm $a_{0}=0$ whenever $m=0$.

For the derivation of the critical number $\kappa_{c}$, we use the eigenfunction

$$
\psi=\sum_{n=-\infty}^{\infty} \frac{a_{n}}{b\left[\left(b^{2}+n^{2}\right)^{\frac{\beta}{2}}-1\right]} \cos \left(b x_{1}+\phi+n x_{2}+\frac{n \pi}{2}\right)
$$


to rewrite (26) as

$$
\begin{aligned}
0= & \sum_{n=-\infty}^{\infty} \frac{2 \kappa^{2}\left(b^{2}+n^{2}\right)^{\frac{\beta}{2}} a_{n}}{b\left[\left(b^{2}+n^{2}\right)^{\frac{\beta}{2}}-1\right]} \cos \left(b x_{1}+\phi+n x_{2}+\frac{n \pi}{2}\right) \\
& +\sum_{n=-\infty}^{\infty} 2 a_{n} \cos x_{2} \sin \left(b x_{1}+\phi+n x_{2}+\frac{n \pi}{2}\right) \\
= & \sum_{n=-\infty}^{\infty}\left(\frac{2 \kappa^{2}\left(b^{2}+n^{2}\right)^{\frac{\beta}{2}} a_{n}}{b\left[\left(b^{2}+n^{2}\right)^{\frac{\beta}{2}}-1\right]}-a_{n-1}+a_{n+1}\right) \cos \left(b x_{1}+\phi+n x_{2}+\frac{n \pi}{2}\right),
\end{aligned}
$$

and so

$$
2 \kappa^{2} d_{n} a_{n}+a_{n+1}-a_{n-1}=0, \quad n \in Z
$$

for

$$
d_{n}=\frac{\left(b^{2}+n^{2}\right)^{\frac{\beta}{2}}}{b\left[\left(b^{2}+n^{2}\right)^{\frac{\beta}{2}}-1\right]} .
$$

This yields

$$
2 \kappa^{2} d_{n} \pm \frac{a_{ \pm(n+1)}}{a_{ \pm n}} \mp \frac{a_{ \pm(n-1)}}{a_{ \pm n}}=0 \text { for } n \geq 0
$$

After an iteration for $n \geq 0$, equation (34) reduces to

$$
\text { (35) } \gamma_{ \pm n} \equiv \frac{a_{ \pm n}}{a_{ \pm(n-1)}}=\frac{ \pm 1}{2 \kappa^{2} d_{n} \pm \frac{a_{ \pm(n+1)}}{a_{ \pm n}}}=\frac{ \pm 1}{2 \kappa^{2} d_{n}+\frac{1}{2 \kappa^{2} d_{n+1}+\frac{1}{\ddots}}} \text { for } n \geq 1
$$

Combining (35) for $n=1$ and (33)-(34) for $n=0$, we have equivalent formulation of the iteration equation $(32)$ :

$$
-d_{0}=\frac{b^{\beta-1}}{1-b^{\beta}}=\frac{1}{2 \kappa^{4} d_{1}+\frac{1}{d_{2}+\frac{1}{2 \kappa^{4} d_{3}+\frac{1}{d_{4}+\frac{1}{\ddots}}}}} .
$$

The function $P(\kappa)$, representing the right-hand side term of $(36)$, is the Stieltjes continued fraction. It follows from [29] or [31, Theorem 28.1] that $P(\kappa)$ uniformly convergent to a positive value and is an analytic function of $\kappa>0$. Upon the observation of $P$ being strictly monotone function of $\kappa$ such that

$$
\lim _{\kappa \rightarrow \infty} P(\kappa)=0, \quad \lim _{\kappa \rightarrow 0} P(\kappa)=\infty,
$$

there exists a unique critical value $\kappa=\kappa_{c}>0$ satisfying (36). Thus for such a critical value $\kappa=\kappa_{c}$, the coefficients $a_{n}$ of the associated eigenfunction $\psi$ in the form of (31) are subject to the expression

$$
a_{n}= \begin{cases}\gamma_{1} \cdots \gamma_{n}, & n \geq 1 \\ 1, & n=0 \\ (-1)^{n} a_{-n}, & n \leq-1\end{cases}
$$


It is readily seen that eigenfunction $\psi$ obtained is smooth. Thus we have

(38)m $\left\{\psi=\sum_{n=-\infty}^{\infty} \frac{a_{n} \cos \left(b x_{1}+\phi+n x_{2}+\frac{n \pi}{2}\right)}{b\left[\left(b^{2}+n^{2}\right)^{\frac{\beta}{2}}-1\right]} \in H_{p}^{1+\beta}\left(\Omega_{b}\right) ;\left(1+M_{\kappa}\right) \psi=0\right\}=1$.

For the spectral simplicity given in (28), it is sufficient to verify the property

(39im $\left\{\psi=\sum_{n=-\infty}^{\infty} \frac{a_{n} \cos \left(b x_{1}+\phi+n x_{2}+\frac{n \pi}{2}\right)}{b\left[\left(b^{2}+n^{2}\right)^{\frac{\beta}{2}}-1\right]} \in H_{p}^{1+\beta}\left(\Omega_{b}\right) ;\left(1+M_{\kappa}\right)^{2} \psi=0\right\}=1$.

We see that the equation $\left(1+M_{\kappa}\right)^{2} \psi=0$ can be written in the form

$$
\psi^{\prime}+M_{\kappa} \psi^{\prime}=0 \text { and } \psi^{\prime}=\psi+M_{\kappa} \psi
$$

or, equivalently,

$$
\begin{aligned}
& \kappa^{2}(-\Delta)^{\frac{\beta}{2}} \psi^{\prime}-\cos x_{2}\left((-\Delta)^{\frac{\beta}{2}}-1\right) \partial_{x_{1}} \psi^{\prime}=0, \\
& \kappa^{2}(-\Delta)^{\frac{\beta}{2}} \psi-\cos x_{2}\left((-\Delta)^{\frac{\beta}{2}}-1\right) \partial_{x_{1}} \psi=\left(\kappa^{2}-\cos x_{2} \partial_{x_{1}}\right](-\Delta)^{\frac{\beta}{2}} \psi^{\prime} .
\end{aligned}
$$

for

$$
\psi^{\prime}=\sum_{n=-\infty}^{\infty} \frac{a_{n}^{\prime} \cos \left(b x_{1}+\phi+n x_{2}+\frac{n \pi}{2}\right)}{b\left[\left(b^{2}+n^{2}\right)^{\frac{\beta}{2}}-1\right]} .
$$

Thus (41)-(42) reduce respectively to the iteration equations

$$
2 \kappa^{2} d_{n} a_{n}^{\prime}+a_{n+1}^{\prime}-a_{n-1}^{\prime}=0
$$

and

$$
2 \kappa^{2} d_{n} a_{n}+a_{n+1}-a_{n-1}=2 \kappa^{2} d_{n} a_{n}^{\prime}+b d_{n+1} a_{n+1}^{\prime}-b d_{n-1} a_{n-1}^{\prime}
$$

for any arbitrary integer $n$. Therefore from the demonstration of (38), it remains to prove that $\psi^{\prime}=0$ or $a_{n}^{\prime} \equiv 0$.

On the contrary, we assume that $a_{n}^{\prime} \neq 0$ and hence satisfies (37). We show a contradiction from this assumption.

Multiplying the $n$th equation of (43) by $(-1)^{n} a_{n}$ and the $n$th equation of (44) by $(-1)^{n} a_{n}^{\prime}$ and then summing the resultant equations respectively, we have

$$
0=\sum_{n=-\infty}^{\infty}(-1)^{n} a_{n}\left[2 \kappa^{2} d_{n} a_{n}^{\prime}+a_{n+1}^{\prime}-a_{n-1}^{\prime}\right]
$$

and

$$
\begin{aligned}
& \sum_{n=-\infty}^{\infty}(-1)^{n} a_{n}^{\prime}\left[2 \kappa^{2} d_{n} a_{n}+a_{n+1}-a_{n-1}\right] \\
& =\sum_{n=-\infty}^{\infty}(-1)^{n} a_{n}^{\prime}\left[2 \kappa^{2} d_{n} a_{n}^{\prime}+b d_{n+1} a_{n+1}^{\prime}-b d_{n-1} a_{n-1}^{\prime}\right] .
\end{aligned}
$$

Rearranging terms in the summations, we see that the right-hand side term of (45) is identical to the left-hand side term of (46). Thus (46) becomes

$$
0=\sum_{n=-\infty}^{\infty}(-1)^{n} 2 \kappa^{2} d_{n} a_{n}^{\prime 2}+\sum_{n=-\infty}^{\infty}(-1)^{n} b a_{n}^{\prime}\left[d_{n+1} a_{n+1}^{\prime}-d_{n-1} a_{n-1}^{\prime}\right],
$$


or

$$
\begin{aligned}
0= & \sum_{n=1}^{\infty}(-1)^{n} 4 \kappa^{2} d_{n} a_{n}^{\prime 2}+2 \kappa^{2} d_{0} a_{0}^{\prime 2} \\
& +\sum_{n=0}^{\infty} 2(-1)^{n} b d_{n+1} a_{n}^{\prime} a_{n+1}^{\prime}-\sum_{n=1}^{\infty} 2(-1)^{n} b d_{n-1} a_{n}^{\prime} a_{n-1}^{\prime},
\end{aligned}
$$

after the use of the identities $a_{-n}^{\prime}=(-1)^{n} a_{n}^{\prime}$ and $d_{n}=d_{-n}$ given by (33) and (37). By the identities

$$
\frac{a_{n}}{a_{n-1}}=\gamma_{n}, \frac{1}{\gamma_{n}}-\gamma_{n+1}=2 \kappa^{2} d_{n}, \gamma_{1}=-d_{0} \kappa^{2}
$$

implied from (32) and (35), the term of (47) including the last two summations is further manipulated as

$$
\begin{aligned}
& \sum_{n=0}^{\infty} 2(-1)^{n} b d_{n+1} a_{n}^{\prime} a_{n+1}^{\prime}-\sum_{n=1}^{\infty} 2(-1)^{n} b d_{n-1} a_{n}^{\prime} a_{n-1}^{\prime} \\
& \quad=\sum_{n=0}^{\infty} 2(-1)^{n} b \frac{d_{n+1} a_{n+1}^{\prime 2}}{\gamma_{n+1}}-\sum_{n=1}^{\infty} 2(-1)^{n} b d_{n-1} a_{n-1}^{\prime 2} \gamma_{n} \\
& =-\sum_{n=1}^{\infty} 2(-1)^{n} b d_{n} a_{n}^{\prime 2} \frac{1}{\gamma_{n}}+\sum_{n=0}^{\infty} 2(-1)^{n} b d_{n} a_{n}^{\prime 2} \gamma_{n+1} \\
& =-\sum_{n=1}^{\infty} 4(-1)^{n} \kappa^{2} b d_{n}^{2} a_{n}^{\prime 2}+2 b d_{0} a_{0}^{\prime 2} \gamma_{1} \\
& =-\sum_{n=1}^{\infty} 4(-1)^{n} \kappa^{2} b d_{n}^{2} a_{n}^{\prime 2}-2 b d_{0}^{2} a_{0}^{\prime 2} \kappa^{2} .
\end{aligned}
$$

On the substitution of (48) into (47), we have

$$
0=\sum_{n=1}^{\infty}(-1)^{n} 4 \kappa^{2}\left(1-b d_{n}\right) d_{n} a_{n}^{\prime 2}+2 \kappa^{2}\left(1-b d_{0}\right) d_{0} a_{0}^{\prime 2}
$$

That is, by employing (33) and removing the factor $4 \kappa^{2}$,

$$
0=\sum_{n=1}^{\infty} \frac{(-1)^{n} d_{n} a_{n}^{\prime 2}}{\left(b^{2}+n^{2}\right)^{\frac{\beta}{2}}-1}-\frac{d_{0} a_{0}^{\prime 2}}{2\left(1-b^{\beta}\right)}
$$

On the other hand, multiplying the $n$th equation of (43) by $a_{n}^{\prime} /\left[4 \kappa^{2}\left(1-b^{\beta}\right)\right]$ and summing the resultant equations yield

$$
0=\sum_{n=-\infty}^{\infty} \frac{d_{n} a_{n}^{\prime 2}}{2\left(1-b^{\beta}\right)}=\sum_{n=1}^{\infty} \frac{d_{n} a_{n}^{\prime 2}}{1-b^{\beta}}+\frac{d_{0} a_{0}^{\prime 2}}{2\left(1-b^{\beta}\right)}
$$


Equation (49) plus equation (50) gives that

$$
\begin{aligned}
0 & =\sum_{n=1}^{\infty} \frac{(-1)^{n} d_{n} a_{n}^{\prime 2}}{\left(b^{2}+n^{2}\right)^{\frac{\beta}{2}}-1}+\sum_{n=1}^{\infty} \frac{d_{n} a_{n}^{\prime 2}}{1-b^{\beta}} \\
& \geq\left(\frac{1}{1-b^{\beta}}-\frac{1}{\left(b^{2}+1\right)^{\frac{\beta}{2}}-1}\right) d_{1} a_{1}^{\prime 2}+\sum_{n=2}^{\infty}\left(\frac{1}{1-b^{\beta}}-\frac{1}{\left(b^{2}+n^{2}\right)^{\frac{\beta}{2}}-1}\right) d_{n} a_{n}^{\prime 2} \\
& >4 \sum_{n=2}^{\infty} \frac{\left(b^{2}+4\right)^{\beta / 2}-2}{\left(1-b^{\beta}\right)\left[\left(b^{2}+n^{2}\right)^{\frac{\beta}{2}}-1\right]} d_{n} a_{n}^{\prime 2},
\end{aligned}
$$

since $\frac{3}{4} \leq b<1$ and $\beta>1$. This leads to a contradiction and hence implies the desired assertion $a_{n}^{\prime} \equiv 0$.

The proof of Lemma 3.1 is completed.

\section{KRASNOSELSKII BIFURCATION THEOREM}

Krasnoselskii bifurcation theorem is fundamental to the proof of Theorem 1.1. For convenience, it is stated in the following.

Theorem 4.1. (Krasnoselskii [23] and Nirenberg [26]) For a Banach space X, a constant value $\kappa_{\text {crit }}>0$ and an open neighborhood $\mathcal{D}$ of the point $\left(0, \kappa_{\text {crit }}\right)$ in the metric space $X \times[0, \infty)$, let $F, M_{\kappa}$ and $N$ be the operators expressed as

$$
F(\psi, \kappa)=\psi+M_{\kappa} \psi+N(\psi, \kappa), \quad(\psi, \kappa) \in \mathcal{D},
$$

and subject to the following conditions:

(i): $F: \mathcal{D} \mapsto X$ is continuous,

(ii): $M_{\kappa}: X \mapsto X$ is linear and $M_{\kappa}: \mathcal{D} \mapsto \mathcal{D}$ is compact and continuous,

(iii): $N: \mathcal{D} \mapsto X$ is nonlinear and compact, $N(0, \kappa) \equiv 0$ and $N(\psi, \kappa)=$ $o\left(\|\psi\|_{X}\right)$ uniformly for $(\psi, \kappa) \in \mathcal{D}$,

(iv): the spectral simplicity condition

$$
\operatorname{dim} \bigcup_{n=1}^{\infty}\left\{\psi \in X ;\left(1+M_{\kappa_{\text {crit }}}\right)^{n} \psi=0\right\}=1
$$

holds true at the critical value $\kappa_{\text {crit }}$.

Then there exists a continuous family $\left(\psi_{\kappa}, \kappa\right) \in \mathcal{D}$, different to the trivial one $(0, \kappa)$, such that

$$
F\left(\psi_{\kappa}, \kappa\right)=0
$$

\section{Proof of Theorem 1.1}

To verify the conditions of Krasnoselskii Theorem, we define the operator

$$
F(\psi, \kappa)=\psi-\kappa^{2}(-\Delta)^{-\frac{\beta}{2}}\left(\kappa^{2}+u \cdot \nabla\right)^{-1} \psi_{0}
$$

Therefore, $F=0$ represents the steady-state equation of Theorem 1.1. Thus we have the desired decomposition form

$$
F(\psi, \kappa)=\psi-\psi_{0}+M_{\kappa}\left(\psi-\psi_{0}\right)+N(\psi, \kappa), \quad \psi_{0}=\sin x_{2}
$$

for the linear operator $M_{\kappa}$ and the nonlinear operator $N$ given in (23) and (24). 
Firstly, for a constant $\tau$ so that $1+2 / p<\tau<\beta$, we introduce the space $X=X_{\phi}$ based on the spectral analysis in Section 3 as

$$
X_{\phi}=\left\{\psi \in H_{p}^{1+\tau}\left(\Omega_{b}\right) ; \psi=\sum_{m=0}^{\infty} \sum_{n=-\infty}^{\infty} a_{m, n} \cos \left(m b x_{1}+m \phi+n x_{2}+\frac{n \pi}{2}\right)\right\} .
$$

Upon the observation of the flow invariance property that

$$
u^{\prime} \cdot \nabla \psi=\left(-\partial_{x_{2}} \psi^{\prime}, \partial_{x_{1}} \psi^{\prime}\right) \cdot \nabla \psi=\sum_{m=0}^{\infty} \sum_{n=-\infty}^{\infty} \tilde{a}_{m, n} \cos \left(m b x_{1}+m \phi+n x_{2}+\frac{n \pi}{2}\right)
$$

for some coefficients $\tilde{a}_{m, n}$ whenever $\psi^{\prime}, \psi \in X_{\phi}$ and $u=\left(-\partial_{x_{2}} \psi, \partial_{x_{1}} \psi\right)$, we have the flow invariance property

$$
(-\Delta)^{-\frac{\beta}{2}}\left[\kappa^{2}+u \cdot \nabla\right]^{-1}: X_{\phi} \mapsto X_{\phi} \text { for } \psi \in X_{\phi}
$$

due to Lemma 2.2. By Lemma 2.2, the choice of the space $X$ also ensures the existence of the critical value $\kappa_{c}>0$ and the spectral simplicity condition (iv)

$$
\operatorname{dim} \bigcup_{n=1}^{\infty}\left\{\psi \in X ;\left(1+M_{\kappa_{c}}\right)^{n} \psi=0\right\}=1
$$

Moreover, we define the metric space

$$
\mathcal{D}=\left\{\psi \in X_{\phi} ;\left\|\nabla^{2} \psi-\nabla^{2} \psi_{0}\right\|_{L_{\infty}} \leq \frac{\left(\kappa_{c}-\epsilon\right)^{4}}{5}\right\} \times\left[\kappa_{c}-\epsilon, \kappa_{c}+\epsilon\right] .
$$

Secondly, we verify the assumptions (i) of Theorem 4.1. By Lemma 2.2, we have the bound

$$
\|F(\psi, \kappa)-\psi\|_{H_{p}^{1+\beta}}=\kappa^{2}\left\|\left(\kappa^{2}+u \cdot \nabla\right)^{-1} \psi_{0}\right\|_{H_{p}^{1}} \leq c\left\|\psi_{0}\right\|_{H_{p}^{1}},
$$

where and in what follows $c$ denotes a generic constant independent of $(\psi, \kappa) \in \mathcal{D}$.

For the continuity of $F$ with respect to $(\psi, \kappa),\left(\psi^{\prime}, \kappa^{\prime}\right) \in \mathcal{D}$ and $u=\left(-\partial_{x_{2}} \psi, \partial_{x_{1}} \psi\right)$ and $u^{\prime}=\left(-\partial_{x_{2}} \psi^{\prime}, \partial_{x_{1}} \psi^{\prime}\right)$, we further use Lemma 2.2 to obtain

$$
\begin{aligned}
\|[ & \left.F\left(\psi^{\prime}, \kappa^{\prime}\right)-F(\psi, \kappa)-\left(\psi^{\prime}-\psi\right)\right] \|_{H_{p}^{\beta}} \\
\leq & \left|\kappa^{\prime 2}-\kappa^{2}\right|\left\|\left(\kappa^{\prime 2}+u^{\prime} \cdot \nabla\right)^{-1} \psi_{0}\right\|_{L_{p}}+\kappa^{2}\left\|\left(\kappa^{2}+u \cdot \nabla\right)^{-1} \psi_{0}-\left(\kappa^{\prime 2}+u^{\prime} \cdot \nabla\right)^{-1} \psi_{0}\right\|_{L_{p}} \\
\leq & c\left|\kappa^{2}-\kappa^{\prime 2}\right|\left\|\psi_{0}\right\|_{L_{p}} \\
& +\kappa^{2}\left\|\left(\kappa^{\prime 2}+u^{\prime} \cdot \nabla\right)^{-1}\left[\kappa^{2}-\kappa^{\prime 2}+\left(u-u^{\prime}\right) \cdot \nabla\right]\left(\kappa^{2}+u \cdot \nabla\right)^{-1} \psi_{0}\right\|_{L_{p}} \\
\leq & c\left|\kappa^{2}-\kappa^{\prime 2}\right|+c\left\|\left[\kappa^{2}-\kappa^{\prime 2}+\left(u-u^{\prime}\right) \cdot \nabla\right]\left(\kappa^{2}+u \cdot \nabla\right)^{-1} \psi_{0}\right\|_{L_{p}} \\
\leq & c\left|\kappa^{2}-\kappa^{\prime 2}\right|+c\left(\left|\kappa^{2}-\kappa^{\prime 2}\right|+\left\|u-u^{\prime}\right\|_{L_{\infty}}\right)\left\|\left(\kappa^{2}+u \cdot \nabla\right)^{-1} \psi_{0}\right\|_{H_{p}^{1}} \\
& \leq c\left|\kappa^{2}-\kappa^{\prime 2}\right|+c\left(\left|\kappa^{2}-\kappa^{\prime 2}\right|+\left\|\nabla \psi-\nabla \psi^{\prime}\right\|_{L_{\infty}}\right)\left\|\psi_{0}\right\|_{H_{p}^{1}} .
\end{aligned}
$$

Therefore, combining (52) and the interpolation inequality

$$
\|\varphi\|_{H_{p}^{1+\tau}} \leq c\|\varphi\|_{H_{p}^{\beta}}^{\beta-\tau}\|\varphi\|_{H_{p}^{1+\beta}}^{1-(\beta-\tau)}
$$

we have $F: \mathcal{D} \mapsto \mathcal{D}$ and the continuity

$$
\left(5 \not \mid D F\left(\psi^{\prime}, \kappa^{\prime}\right)-F(\psi, \kappa)-\left(\psi^{\prime}-\psi\right)\right] \|_{H_{p}^{1+\tau}} \leq c\left(\left|\kappa^{2}-\kappa^{\prime 2}\right|+\left\|\psi-\psi^{\prime}\right\|_{H_{\infty}^{2}}\right)^{\beta-\tau} .
$$


Thirdly, the validity of the condition (ii) of Theorem 4.1 with respect to the linear operator $M_{\kappa}$ is obtained in the similar manner. It follows from Lemma 2.2 that for $(\kappa, \psi),\left(\kappa^{\prime}, \psi^{\prime}\right) \in \mathcal{D}$,

$$
\begin{aligned}
& \left\|M_{\kappa} \psi-M_{\kappa^{\prime}} \psi^{\prime}\right\|_{H_{p}^{1+\beta}} \\
& \quad \leq\left\|\left(\kappa^{2}+u_{0} \cdot \nabla\right)^{-1}\left(u \cdot \nabla \psi_{0}-u^{\prime} \cdot \nabla \psi_{0}\right)\right\|_{H_{p}^{1}}+\left\|\left[\left(\kappa^{2}+u_{0} \cdot \nabla\right)^{-1}-\left(\kappa^{\prime 2}+u_{0} \cdot \nabla\right)^{-1}\right] u \cdot \nabla \psi_{0}\right\|_{H_{p}^{1}} \\
& \quad \leq c\left\|u \cdot \nabla \psi_{0}-u^{\prime} \cdot \nabla \psi_{0}\right\|_{H_{p}^{1}}+\left|\kappa^{2}-\kappa^{\prime 2}\right|\left\|\left(\kappa^{2}+u_{0} \cdot \nabla\right)^{-1}\left(\kappa^{\prime 2}+u_{0} \cdot \nabla\right)^{-1} u^{\prime} \cdot \nabla \psi_{0}\right\|_{H_{p}^{1}} \\
& \quad \leq c\left\|\psi-\psi^{\prime}\right\|_{H_{p}^{2}}+c\left|\kappa^{2}-\kappa^{\prime 2}\right|\left\|\psi^{\prime}\right\|_{H_{p}^{2}} .
\end{aligned}
$$

This gives the required compactness and continuity property

$$
\left\|M_{\kappa} \psi-M_{\kappa^{\prime}} \psi^{\prime}\right\|_{H_{p}^{1+\beta}} \leq c\left|\kappa-\kappa^{2}\right|+c\left\|\psi-\psi^{\prime}\right\|_{H_{p}^{1+\tau}}
$$

since the imbedding $H_{p}^{1+\beta} \hookrightarrow H_{p}^{1+\tau}$ is compact.

Finally, to verify the assumptions (iii), we notice that the compactness and continuity of the operator

$$
N(\psi, \kappa)=F(\psi, \kappa)-\psi+\psi_{0}-\kappa M_{\kappa}\left(\psi-\psi_{0}\right)
$$

has been given by (54) and (55) since the imbedding $H_{p}^{1+\tau}\left(\Omega_{b}\right) \hookrightarrow H_{\infty}^{2}\left(\Omega_{b}\right)$ is compact due to the condition $1+2 / p<\tau$.

It now remains to prove the nonlinear property. To do so, we apply the identities

$$
(-\Delta)^{-\frac{\beta}{2}} \kappa^{2}\left(\kappa^{2}+u_{0} \cdot \nabla\right)^{-1} \psi_{0}=(-\Delta)^{-\frac{\beta}{2}} \psi_{0}=\psi_{0}
$$

and

to the operator

$$
u_{0} \cdot \nabla\left(\psi-\psi_{0}\right)=-\left(u-u_{0}\right) \cdot \nabla \psi_{0}
$$

$N=-\kappa^{2}(-\Delta)^{-\frac{\beta}{2}}\left(\kappa^{2}+u \cdot \nabla\right)^{-1} \psi_{0}+\psi_{0}+(-\Delta)^{-\frac{\beta}{2}}\left(\kappa^{2}+u_{0} \cdot \nabla\right)^{-1} u_{0} \cdot \nabla\left(\psi-\psi_{0}\right)$

to obtain that

$$
\begin{aligned}
(-\Delta)^{\frac{\beta}{2}} N(\psi, \kappa)= & \kappa^{2}\left(\kappa^{2}+u \cdot \nabla\right)^{-1}\left(u-u_{0}\right) \cdot \nabla\left(\kappa^{2}+u_{0} \cdot \nabla\right)^{-1} \psi_{0} \\
& -\left(\kappa^{2}+u_{0} \cdot \nabla\right)^{-1}\left(u-u_{0}\right) \cdot \nabla \psi_{0} \\
= & {\left[\left(\kappa^{2}+u \cdot \nabla\right)^{-1}-\left(\kappa^{2}+u_{0} \cdot \nabla\right)^{-1}\right]\left(u-u_{0}\right) \cdot \nabla \psi_{0} } \\
= & (\kappa+u \cdot \nabla)^{-1}\left(u-u_{0}\right) \cdot \nabla\left(\kappa+u_{0} \cdot \nabla\right)^{-1}\left(u-u_{0}\right) \cdot \nabla \psi_{0} .
\end{aligned}
$$

This together with Lemma 2.2 implies

$$
\begin{aligned}
\|N(\psi, \kappa)\|_{H_{p}^{\beta}} & \leq c\left\|\left(u-u_{0}\right) \cdot \nabla\left(\kappa^{2}+u_{0} \cdot \nabla\right)^{-1}\left(u-u_{0}\right) \cdot \nabla \psi_{0}\right\|_{L_{p}} \\
& \leq c\left\|\nabla \psi-\nabla \psi_{0}\right\|_{L_{\infty}}\left\|\nabla\left(\kappa^{2}+u_{0} \cdot \nabla\right)^{-1}\left(u-u_{0}\right) \cdot \nabla \psi_{0}\right\|_{L_{p}} \\
& \leq c\left\|\nabla \psi-\nabla \psi_{0}\right\|_{L_{\infty}}\left\|\psi-\psi_{0}\right\|_{H_{p}^{2}} \leq c\left\|\psi-\psi_{0}\right\|_{H_{p}^{2}}^{2}
\end{aligned}
$$

and

$$
\begin{aligned}
\|N(\psi, \kappa)\|_{H_{p}^{1+\beta}} & =\left\|\left[\left(\kappa^{2}+u \cdot \nabla\right)^{-1}-\left(\kappa^{2}+u_{0} \cdot \nabla\right)^{-1}\right]\left(u-u_{0}\right) \cdot \nabla \psi_{0}\right\|_{H_{p}^{1}} \\
& \leq c\left\|\left(u-u_{0}\right) \cdot \nabla \psi_{0}\right\|_{H_{p}^{1}} \\
& \leq c\left\|\psi-\psi_{0}\right\|_{H_{p}^{2}} .
\end{aligned}
$$

Applying the interpolation inequality (53) to the estimates (56) and (57), we have the desired inequality

$$
\|N(\psi, \kappa)\|_{H_{p}^{1+\tau}} \leq c\left\|\psi-\psi_{0}\right\|_{H_{p}^{2}}^{2(\beta-\tau)+1-(\beta-\tau)} \leq c\left\|\psi-\psi_{0}\right\|_{H_{p}^{1+\tau}}^{1+(\beta-\tau)} .
$$


The bifurcation assertion of Theorem 1.1 is thus follows from Theorem 4.1. The upper bound (4) of the critical value $\kappa_{c}$ is from the inequality

$$
\frac{b^{\beta-1}}{1-b^{\beta}} \leq \frac{1}{2 \kappa_{c}^{4} \frac{\left(1+b^{2}\right)^{\frac{\beta}{2}}}{b\left[\left(1+b^{2}\right)^{\frac{\beta}{2}}-1\right]}} \leq \frac{\beta b^{3}}{4 \kappa_{c}^{4}},
$$

which is derived from (36). The proof of Theorem 1.1 is complete.

Acknowledgment. This research is partially supported by NSFC of China (Grant No. 11571240).

\section{References}

\section{REFERENCES}

[1] L. A. Caffarelli, A. Vasseur, Drift diffusion equations with fractional diffusion and the quasigeostrophic equation, Annal. Math. 171 (2010), 1903-1930.

[2] D. Chae, J. Lee, Global well-posedness in the super-critical dissipative quasi-geostrophic equations, Comm. Math. Phys. 233 (2003), 297-311.

[3] J. Chen, Z. -M. Chen, Commutator estimate in terms of partial derivatives of solutions for the dissipative quasi-geostrophic equation, J. Math. Anal. Appl. 444 (2016), 755-767.

[4] Q. Chen, C. Miao, Z. Zhang, A new Bernstein's inequality and the 2D dissipative quasigeostrophic equation, Comm. Math. Phys., 271 (2007), 821-838.

[5] Z. -M. Chen, Steady-state bifurcation analysis of a strong atmospheric vorticity equation, J. Math. Anal. Appl. 431 (2015), 1-21.

[6] Z. -M. Chen, M. Ghil, E. Simonnet, S. Wang, Hopf bifurcation in quasi-geostrophic channel flow, SIAM J. Appl. Math. 64 (2003), 343-368.

[7] Z. -M. Chen, W. G. Price, Stability and instability analyses of the dissipative quasigeostrophic equation, Nonlinearity 21 (2008), 765-782.

[8] Z.-M. Chen, X. Xiong, Equilibrium states of the Charney-DeVore quasigeostrophic equation in mid-latitude atmosphere, J. Math. Anal. Appl. (2016), http://dx.doi.org/10.1016/j.jmaa.2016.07.021

[9] P. Constantin, G. Iyer, J. Wu, Global regularity for a modified critical dissipative quasigeostrophic equation, Indiana Univ. Math. J. 57 (2008), 2681-2697.

[10] P. Constantin, A. Majda, E. Tabak, Formation of strong fronts in the 2D quasi-geostrophic thermal active scalar, Nonlinearity 7 (1994), 1495-1533

[11] P. Constantin, J. Wu, Behavior of solutions of 2D quasi-geostrophic equations, SIAM J. Math. Anal. 30 (1999), 937-948.

[12] P. Constantin, J.Wu, Regularity of Hölder continuous solutions of the supercritical quasigeostrophic equation, Ann. I. H. Poincaré-AN 25 (2008), 1103-1110.

[13] A. Córdoba, D. Córdoba, A maximum principle applied to quasi-geostrophic equations, Commun. Math. Phys. 249 (2004), 511-528.

[14] R. Danchin, Density-dependent incompressible viscous fluids in critical spaces, Proc. Roy. Soc. Edinburgh 133A (2003), 1311-1334.

[15] H. Dong, N. Pavlović, A regularity criterion for the dissipative quasi-geostrophic equations, Ann. I. H. Poincaré-AN 26 (2009), 1607-1619.

[16] I. M. Held, R. T. Pierrehumbert, S. T. Garner, K. L. Swanson, 1995 Surface quasi-geostrophic dynamics, J. Fluid Mech. 282 (1995), 1-20.

[17] G. R. Ierley, V. A. Sheremet, Multiple solutions and advection-dominated flows in the winddriven circulation, Part I: Slip, J. Marine Res. 53 (1995), 703-737.

[18] N. Ju, The maximum principle and the global attractor for the dissipative 2D quasigeostrophic equations, Comm. Math. Phys. 255 (2005), 161-181.

[19] A. Kiselev, Some recent results on the critical surface quasi-geostrophic equation: A review, Proceedings of Symposia in Applied Math. 67.1 (2009), 105-121.

[20] A. Kiselev, Regularity and blow up for active scalars, Math. Model. Nat. Phenom. 5 (2010), 225-255. 
[21] A. Kiselev, F. Nazarov, A. Volberg, Global well-posedness for the critical 2d dissipative quasi-geostrophic equation, Invent. Math. 167 (2007), 445-453.

[22] T. Kato, G. Ponce, Commutator estimates and the Euler and NavierStokes equations, Comm. Pure Appl. Math. 41 (1988), 891-907.

[23] M. A. Krasnoselskii, Topological Methods in the Theory of Nonlinear Integral Equations, New York, Macmillan, 1964.

[24] T. Ma, S. Wang, Bifurcation Theory and Applications, World Scientific, Singapore, 2005.

[25] L. D. Meshalkin, Ya G. Sinai, Investigation of the stability of a stationary solution of a system of equations for the plane movement of an incompressible viscous fluid, J. Math. Mech. 19 (1961), 1700-1705.

[26] L. Nirenberg, Topics in Nonlinear Functional Analysis, New York, Courant Institute, 1974.

[27] J. Pedlosky, Geophysical Fluid Dynamics, Springer, New York, 1987.

[28] M. E. Schonbek, T. P. Schonbek, Asymptotic behavior to dissipative quasi-geostrophic flows, SIAM J. Math. Anal. 35 (2003), 357-375.

[29] J. J. Stieltjes, Recherches Sur les Fractions Continues, Ann. Fac. Sci. Toulouse 8 (1894), $1-122$.

[30] G. Veronis, An analysis of wind-driven ocean circulation with a limited number of Fourier components, J. Atmos. Sci. 20 (1963), 577-593.

[31] H. S. Wall, Analytic Theory of Continued Fractions, New York, D.Van Nostrand, 1948.

[32] J. Wu, Quasi-geostrophic-type equations with initial data in Morrey spaces, Nonlinearity 10 (1997), 1409-1420.

[33] J. Wu, Global solutions of the 2d dissipative quasi-geostrophic equation in Besov spaces, SIAM J. Math. Anal. 36 (2005), 1014-1030.

[34] K. Yamazaki, On the regularity criteria of a surface quasi-geostrophic equation, Nonlinear Anal.: TMA 75 (2012), 4950-4956.

[35] V. I. Yudovich, Example of the generation of a secondary stationary or periodic flow when there is loss of stability of the laminar flow of a viscous incompressible fluid, J. Appl. Math. Mech. 29 (1965), 453-467.

School of Mathematics and Statistics, Shenzhen University, Shenzhen 518052, China

Ship Science, University of Southampton, Southampton SO17 1BJ, UK

E-mail address, corresponding address: zhimin@soton.ac.uk 